In Proc. IEEE Int. Conf. Acoust. Speech Signal Process., Atlanta, GA, 1996, vol. 3, pp. 1415-1418.

\title{
INTERACTIVE WAVELET PROCESSING AND TECHNIQUES APPLIED TO DIGITAL MAMMOGRAPHY
}

\author{
Iztok Koren ${ }^{1} \quad$ Andrew Laine Fred Taylor $^{2} \quad$ Michael Lewis ${ }^{3}$ \\ ${ }^{1}$ Department of Electrical and Computer Engineering \\ ${ }^{2}$ Computer and Information Science and Engineering Department \\ ${ }^{1,2}$ University of Florida, Gainesville, FL 32611 \\ ${ }^{3}$ Athena Group, Inc., Gainesville, FL 32603
}

\begin{abstract}
We present an interactive scheme for processing of digital mammograms relying upon a steerable dyadic wavelet transform. Coefficients of the translation and rotationinvariant transform are interactively processed before an inverse transform is applied. Analysis is carried out at dyadic scales and along arbitrary orientations. Local orientation is computed at each level of scale and spatial position and formulated into criteria for including or excluding specific orientations for contrast enhancement and enhancing locally radiating structures. Transform coefficients that were selected for contrast enhancement are modified by a piecewise linear enhancement function. The presented scheme is flexible enough to enable efficient position, scale, and orientation based interactive processing and analysis.
\end{abstract}

\section{INTRODUCTION}

In mammography, early detection of breast cancer relies upon the ability to distinguish between malignant and benign mammographic features. The complexity of mammographic images and the subtlety of malignancies can present a challenge even to expert radiologists. In addition to dealing with barely visible mammographic features, human observers sometimes simply overlook abnormalities. Such mistakes affect the number of false-negative cases considerably. Computer processing of digital mammograms can assist radiologists to reach a correct diagnosis more consistently. A variety of computer based techniques have been reported in almost three decades of research [1]. The advent of direct digital mammography devices has made digital image processing techniques more attractive for screening.

In [2], separable and nonseparable multiscale transforms were used for adaptive contrast enhancement of mammographic features and the results were compared with traditional image enhancement techniques. Measuring the local contrast of known mammographic features showed a significant advantage of multiscale techniques over traditional approaches. In [3], contrast enhancement was achieved within a discrete dyadic wavelet transform framework. It was shown that wavelets which are equal to a second derivative of an odd degree spline do not introduce artifacts when combined with a piecewise linear enhancement function. Furthermore, it was demonstrated that traditional unsharp masking is included in a discrete dyadic wavelet transform scheme for contrast enhancement.

Here, we build on our previous work by using a discrete steerable dyadic wavelet transform with a steerable wavelet equal to a second derivative of a circularly symmetric odd degree spline. Steerability allows analysis along arbitrary orientations and in addition to being translation-invariant, the transform is rotation-invariant. The analysis of orientation was proven successful for the detection of breast masses. In [4] excellent sensitivity for using an orientation based spiculated lesion detection algorithm was reported. Here, we gave the radiologist the freedom to interactively change the parameters and method of enhancement. A piecewise linear enhancement function [5] can be used either to modify the discrete steerable dyadic wavelet transform coefficients uniformly, or to modify only those coefficients that are oriented within a specified interval of orientations. Orientation of coefficients is based on the local oriented energy determined from the transform coefficients and their Hilbert transform counterparts. Local orientation analysis is also possible: local statistics on the similarity of coefficient orientation was carried out and only those coefficients with large differences in edge orientation were enhanced.

This paper is organized as follows. Section 2 formulates a discrete steerable dyadic wavelet transform. Next, interactive wavelet techniques are described in Section 3. Preliminary results are then shown in Section 4. Finally, Section 5 presents a brief summary.

\section{A DISCRETE STEERABLE DYADIC}

A discrete steerable dyadic wavelet transform [6] incorporates the concept of steerability [7] into a discrete dyadic wavelet transform framework [8].

Steerable functions are functions whose arbitrary rotation can be synthesized from a linear combination of basis functions. A function is steerable if it has a finite number of terms in its Fourier series expansion of its polar angle [7]. Functions that are not steerable can be approximated with steerable functions [9].

A discrete dyadic wavelet transform is computed at dyadic scales and integer translations. In [8] they used a wavelet which was equal to a first derivative of an odd degree spline. A filter bank implementation associated with a wavelet being a second derivative of an odd degree spline was reported in [5]. In two dimensions, separable wavelets were constructed as tensor products of onedimensional wavelets and smoothing functions. Thus the resulting two-dimensional discrete dyadic wavelet transform is translation, but not rotation-invariant. We construct two-dimensional steerable wavelets and use them in a translation-invariant and rotation-invariant discrete steerable dyadic wavelet transform.

We define a steerable dyadic wavelet transform of a function $f(x, y) \in L^{2}\left(\boldsymbol{R}^{2}\right)$ at a scale $2^{m}, m \in Z$, as

$$
W_{2^{m}}^{\theta_{k}} f(x, y)=\int_{-\infty}^{\infty} \int_{-\infty}^{\infty} f(u, v) \psi_{2^{m}}^{\theta_{k}}(x-u, y-v) d u d v
$$

where $\psi_{2^{m}}(x, y)=2^{-2 m} \psi\left(2^{-m} x, 2^{-m} y\right), \psi(x, y)$ is a steerable wavelet that can be steered with $K$ basis functions, $\psi_{2^{m}}^{\theta_{k}}(x, y)$ denotes $\psi_{2^{m}}(x, y)$ rotated by $\theta_{k}$, and $\left\{\theta_{k}\right\}=\left\{\frac{k-1}{K} \pi\right\}$ with $k \in\{1,2, \ldots, K\}$. 
If (nonunique) functions $\chi_{2_{m}}^{\theta_{k}}(x, y)$ are chosen such that their Fourier transforms satisfy

$$
\sum_{m=-\infty}^{\infty} \sum_{k=1}^{K} \hat{\psi}^{\theta k}\left(2^{m} \omega_{x}, 2^{m} \omega_{y}\right) \hat{\chi}^{\theta k}\left(2^{m} \omega_{x}, 2^{m} \omega_{y}\right)=1
$$

the function $f(x, y)$ may be reconstructed from its steerable dyadic wavelet transform by

$f(x, y)=\sum_{m=-\infty}^{\infty} \sum_{k=1}^{K} \int_{-\infty}^{\infty} \int_{-\infty}^{\infty} W_{2^{m}}^{\theta_{k}} f(x, y) \chi_{2^{m}}^{\theta_{k}}(x-u, y-v) d u d v$.

For discrete signal processing we normalize the finest scale to 1 , set the coarsest scale to $2^{M}$ [8], and introduce a smoothing function $\phi(x, y)$ such that

$$
\left|\hat{\phi}\left(\omega_{x}, \omega_{y}\right)\right|^{2}=\sum_{m=1}^{\infty} \sum_{k=1}^{K} \hat{\psi}^{\theta_{k}}\left(2^{m} \omega_{x}, 2^{m} \omega_{y}\right) \hat{\chi}^{\theta_{k}}\left(2^{m} \omega_{x}, 2^{m} \omega_{y}\right)
$$

Next, we derive a filter bank implementation of a discrete steerable dyadic wavelet transform with an analyzing function equal to a second derivative of an isotropic odd degree spline. Let us use polar coordinates with $\omega_{r}=\sqrt{\omega_{x}^{2}+\omega_{y}^{2}}$ and $\omega_{\phi}=\arg \left(\omega_{x}, \omega_{y}\right)$. Analogous to [8] we write the Fourier transform of a real smoothing function as

$$
\hat{\phi}\left(\omega_{r}\right)=\prod_{m=1}^{\infty} H\left(2^{-m} \omega_{r}\right)
$$

Using the class of filters proposed in [5]

$$
H\left(\omega_{r}\right)=\left(\cos \left(\frac{\omega_{r}}{2}\right)\right)^{2 n}, \quad n \in N
$$

and relation $\prod_{m=1}^{\infty} \cos \left(2^{-m} \omega\right)=\frac{\sin \omega}{\omega}$, we obtain

$$
\hat{\phi}\left(\omega_{r}\right)=\left(\frac{\sin \left(\frac{\omega_{r}}{2}\right)}{\frac{\omega_{r}}{2}}\right)^{2 n}
$$

Equation (2) implies

$$
\hat{\phi}\left(2 \omega_{r}\right)=H\left(\omega_{r}\right) \hat{\phi}\left(\omega_{r}\right) .
$$

Let us choose

$$
\hat{\psi}\left(2 \omega_{r}, \omega_{\phi}\right)=G\left(\omega_{r}, \omega_{\phi}\right) \hat{\phi}\left(\omega_{r}\right)
$$

and

$$
\hat{\chi}\left(2 \omega_{r}, \omega_{\phi}\right)=K\left(\omega_{r}, \omega_{\phi}\right) \hat{\phi}\left(\omega_{r}\right)
$$

To obtain a second derivative wavelet we select

$$
G\left(\omega_{r}, \omega_{\phi}\right)=-16\left(\sin \left(\frac{\omega_{r}}{2}\right)\right)^{2} \cos ^{2}\left(\omega_{\phi}\right)
$$

Using Equation (7) with Equation (5), we then obtain

$$
\hat{\psi}\left(\omega_{r}, \omega_{\phi}\right)=-\omega_{r}^{2}\left(\frac{\sin \left(\frac{\omega_{r}}{4}\right)}{\frac{\omega_{r}}{4}}\right)^{2 n+2} \cos ^{2}\left(\omega_{\phi}\right) .
$$

The obtained wavelet is a second derivative of a circularly symmetric spline of degree $2 n+1$ in the direction of $x$-axis and is shown in Figure 1 for $n=2$.

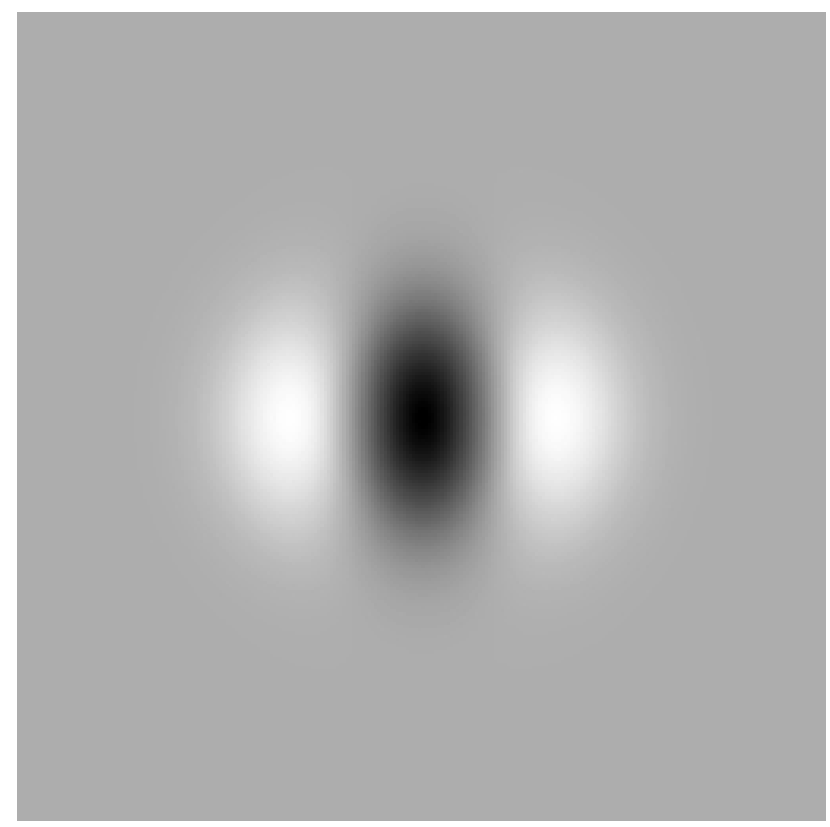

Figure 1. A steerable wavelet equal to a second derivative of a circularly symmetric quintic spline.

Both filter $G\left(\omega_{r}, \omega_{\phi}\right)$ and wavelet $\hat{\psi}\left(\omega_{r}, \omega_{\phi}\right)$ are steerable with three basis functions:

$$
\begin{aligned}
G\left(\omega_{r}, \omega_{\phi}-\theta\right) & =\sum_{k=1}^{3} a_{k}(\theta) G\left(\omega_{r}, \omega_{\phi}-\theta_{k}\right) \\
\hat{\psi}\left(\omega_{r}, \omega_{\phi}-\theta\right) & =\sum_{k=1}^{3} a_{k}(\theta) \hat{\psi}\left(\omega_{r}, \omega_{\phi}-\theta_{k}\right),
\end{aligned}
$$

where the interpolation functions were $a_{k}(\theta)=\frac{1}{3}(1+$ $\left.2 \cos \left(2\left(\theta-\theta_{k}\right)\right)\right)$ with $\theta_{k}=(k-1) \frac{\pi}{3}$.

Computing Equation (1) for the finest two scales shows that

$\sum_{k=1}^{3} \hat{\psi}\left(2 \omega_{r}, \omega_{\phi}-\theta_{k}\right) \hat{\chi}\left(2 \omega_{r}, \omega_{\phi}-\theta_{k}\right)=\left|\hat{\phi}\left(\omega_{r}\right)\right|^{2}-\left|\hat{\phi}\left(2 \omega_{r}\right)\right|^{2}$.

Inserting Equations (4), (5), and (6) into Equation (9) results in a relation between filter frequency responses

$$
\left|H\left(\omega_{r}\right)\right|^{2}+\sum_{k=1}^{3} G\left(\omega_{r}, \omega_{\phi}-\theta_{k}\right) K\left(\omega_{r}, \omega_{\phi}-\theta_{k}\right)=1 .
$$

The remaining filter frequency response $K\left(\omega_{r}, \omega_{\phi}\right)$ can now be determined by inserting Equations (3) and (7) into Equation (10) and using the relation $\sum_{k=1}^{3} \cos ^{4}\left(\omega_{\phi}-\theta_{k}\right)=$ $\frac{9}{8}:$

$$
K\left(\omega_{r}, \omega_{\phi}\right)=-\frac{1}{18} \sum_{l=0}^{2 n-1}\left(\cos \left(\frac{\omega_{r}}{2}\right)\right)^{2 l} \cos ^{2}\left(\omega_{\phi}\right) .
$$

Filter $K\left(\omega_{r}, \omega_{\phi}\right)$ is steerable with the same interpolation functions $a_{k}(\theta)$ as $G\left(\omega_{r}, \omega_{\phi}\right)$ and $\hat{\psi}\left(\omega_{r}, \omega_{\phi}\right)$ :

$$
K\left(\omega_{r}, \omega_{\phi}-\theta\right)=\sum_{k=1}^{3} a_{k}(\theta) K\left(\omega_{r}, \omega_{\phi}-\theta_{k}\right) .
$$


Figure 2 shows basic modules of the filter bank implementation of a discrete steerable dyadic wavelet transform. After the decomposition module with $m=0$ is applied to the original signal, the module is iterated for $m \in[1, M]$ at the output of $H\left(2^{m} \omega_{r}\right)$. The structure of the reconstruction part simply mirrors the one of the decomposition part. Such an implementation shares some properties with steerable pyramids $[10,11,12]$, but is more overcomplete.

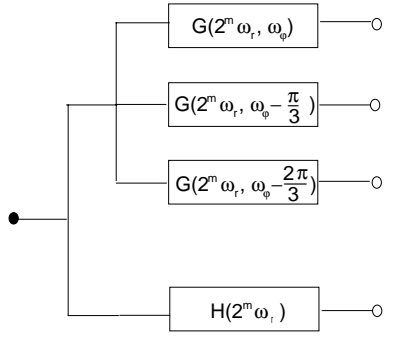

(a)

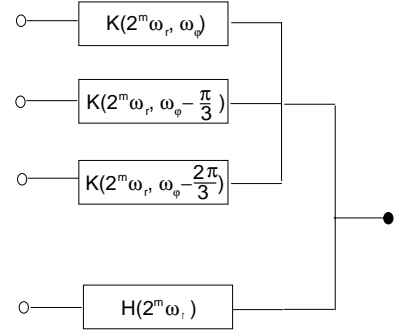

(b)
Figure 2. Basic decomposition (a) and reconstruction (b) modules of the filter bank implementation of a discrete steerable dyadic wavelet transform.

\section{INTERACTIVE PROCESSING
TECHNIQUES}

Contrast enhancement within the discrete dyadic wavelet transform framework was achieved by applying a piecewise linear enhancement function to transform coefficients [13]. Here, an enhancement function was applied to discrete steerable dyadic wavelet transform coefficients. After the forward transform was performed, normalized coefficients were modified by

$$
E(x)= \begin{cases}x-(K-1) T & \text { if } x<-T \\ K x & \text { if }|x| \leq T \\ x+(K-1) T & \text { if } x>T\end{cases}
$$

at each dyadic scale and orientation $\theta_{k}$ separately. The desirable scales and orientations were selected for processing with function $E(x)$ from Equation (11). Figure 3 shows the enhancement function for parameter values $K=20$ and $T=1$.

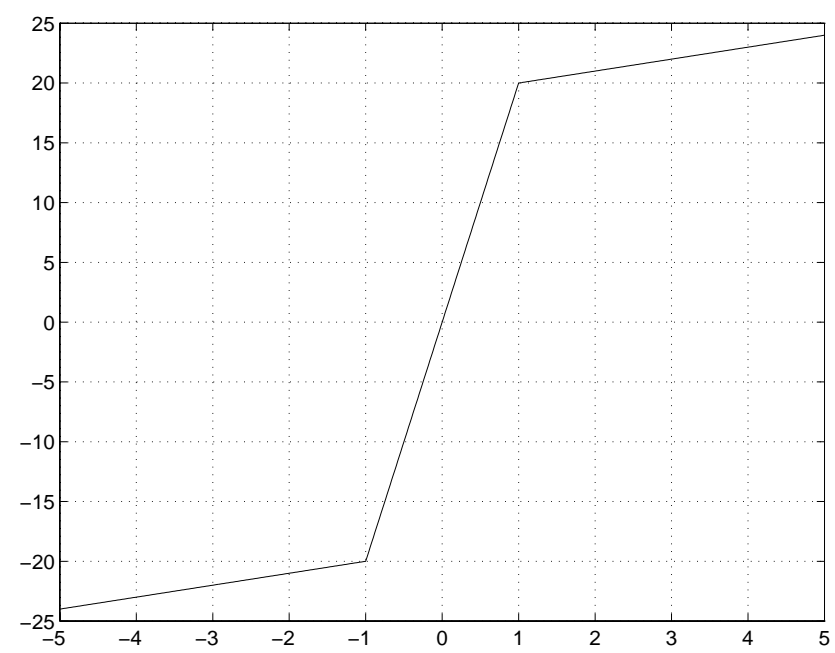

Figure 3. An enhancement function (Equation 11 with $K=20$ and $T=1$ ).
Local orientation is defined as the angle that maximizes the local oriented energy, obtained from Hilbert transform pairs of the discrete steerable dyadic wavelet transform $\mathrm{CO}^{-}$ efficients. The Hilbert transforms of the filter $G\left(\omega_{r}, \omega_{\phi}\right)$ and the wavelet $\hat{\psi}\left(\omega_{r}, \omega_{\phi}\right)$ are given by

$$
G_{H}\left(\omega_{r}, \omega_{\phi}\right)=-j \operatorname{sgn}\left(\cos \left(\omega_{\phi}\right)\right) G\left(\omega_{r}, \omega_{\phi}\right)
$$

and

$$
\hat{\psi}_{H}\left(\omega_{r}, \omega_{\phi}\right)=-j \operatorname{sgn}\left(\cos \left(\omega_{\phi}\right)\right) \hat{\psi}\left(\omega_{r}, \omega_{\phi}\right),
$$

respectively, where

$$
\operatorname{sgn}(x)= \begin{cases}1 & \text { if } x \geq 0 \\ -1 & \text { if } x<0 .\end{cases}
$$

The transform coefficients with local orientation within a chosen interval of orientations can be included or excluded in processing by the enhancement function. Since in a normal mammogram the structure radiates from the nipple to the chest wall, this type of processing can be used to identify areas with locally radiating structures.

Another technique that we are using is similar to the one described in [4], but used here in a multiscale steerable framework. 1-norm of differences between local orientation and average orientation is computed within a sliding window and used as a measure of orientation nonuniformity within the window. Soft thresholding as a function of orientation nonuniformity measure is used on the transform coefficients at each dyadic scale independently.

\section{RESULTS}

An original mammographic image containing a spicular mass is shown in Figure 4. Figure 5 shows the result of applying the enhancement from Equation (11) with pa-

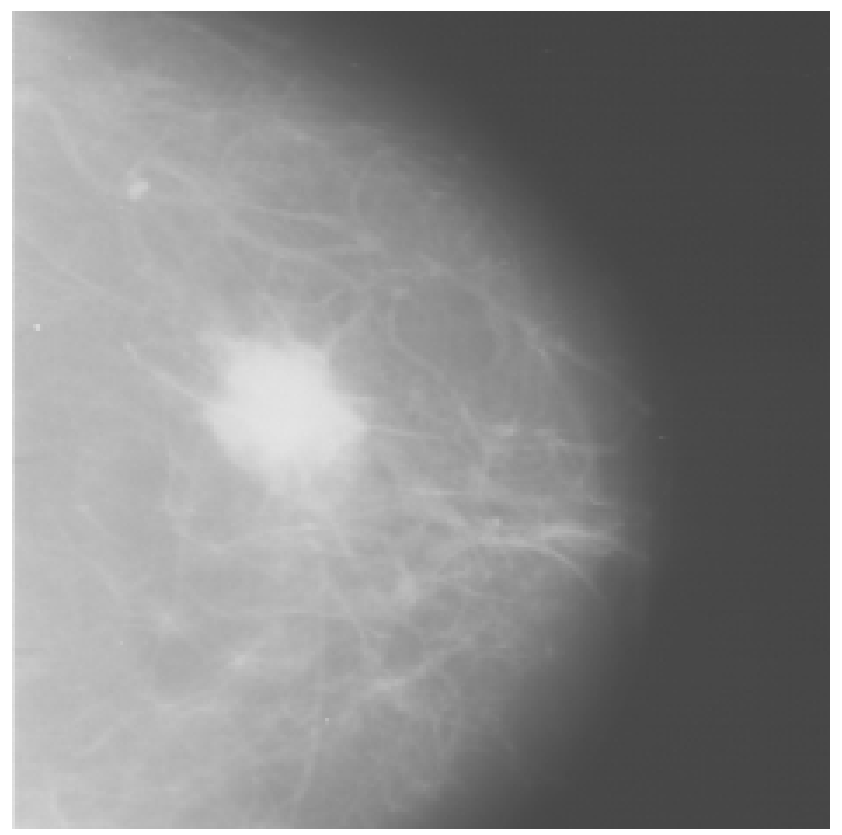

Figure 4. An original mammographic image containing a spicular mass.

rameters $K=20$ and $T=0.1$ across five dyadic scales (i.e., $M=4$ ). The result of excluding the discrete steerable dyadic wavelet transform coefficients with orientation $\frac{\pi}{3}$ from processing with the enhancement function is shown in Figure 6 (the enhancement was performed with the same set of parameters as shown in Figure 5). 


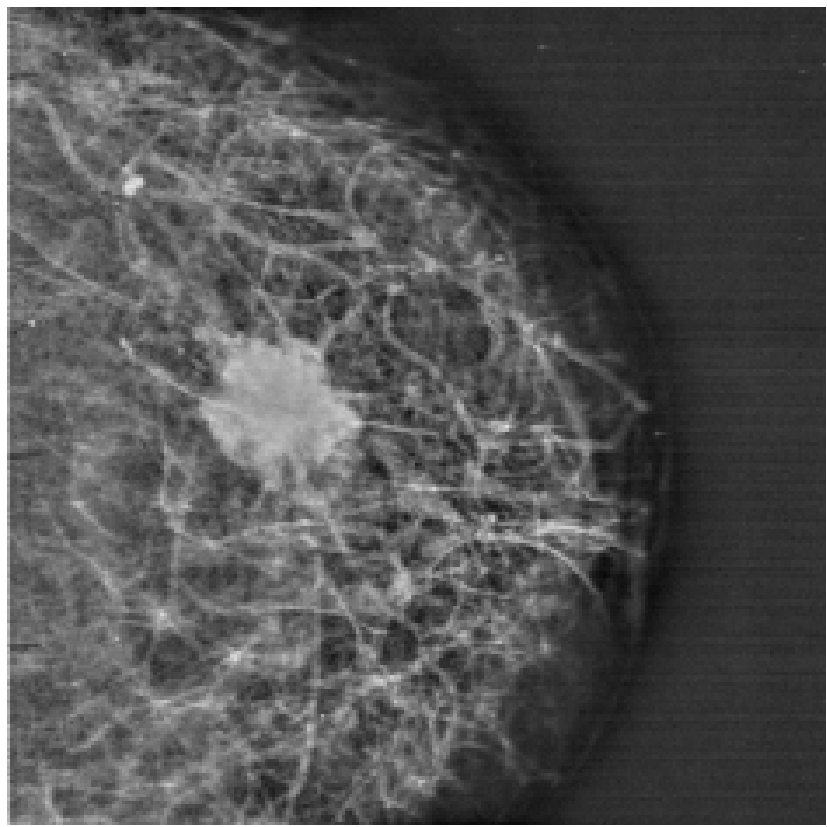

Figure 5. An enhanced image using the enhancement function defined by Equation (11) with parameters $K=20, T=0.1$, and $M=4$.

\section{CONCLUSION}

In this paper we have described a scheme for interactive processing of digital mammograms. Overcompleteness of a discrete steerable dyadic wavelet transform was exploited for an efficient interactive processing across dyadic scales and along arbitrary orientations. Translation and rotationinvariance of the transform are both desirable to prevent unwanted artifacts. A second derivative wavelet compares favorably with our previous experimental results using a discrete dyadic wavelet transform $([5,13,3])$.

The multiscale nature of the presented scheme makes dealing with various-sized malignancies possible, while orientation analysis enables better control over "structured noise" (i.e., normal structures superimposed on features of interest) which can make the reliable interpretation of mammograms difficult.

\section{ACKNOWLEDGMENT}

This work was supported in part by the Whitaker Foundation and the U.S. Army Medical Research and Development Command, Grant No. DAMD17-93-J-3003.

\section{REFERENCES}

[1] C. J. Vyborny and M. L. Giger, "Computer vision and artificial intelligence in mammography," $A J R$, vol. 162 , pp. 699-708, 1994.

[2] A. F. Laine, S. Schuler, J. Fan, and W. Huda, "Mammographic feature enhancement by multiscale analysis," IEEE Trans. Med. Imaging, vol. MI-13, pp. 725740, 1994

[3] J. Fan and A. Laine, "Contrast enhancement of digital mammography," to appear as a chapter within Wavelet Applications in Medicine and Biology, Boca Raton, FL: CRC Press, 1996.

[4] W. P. Kegelmeyer, J. M. Pruneda, P. D. Bourland, A. Hillis, M. V. Riggs, and M. L. Nipper, "Computeraided mammographic screening for spiculated lesions," Radiology, vol. 191, pp. 331-337, 1994.

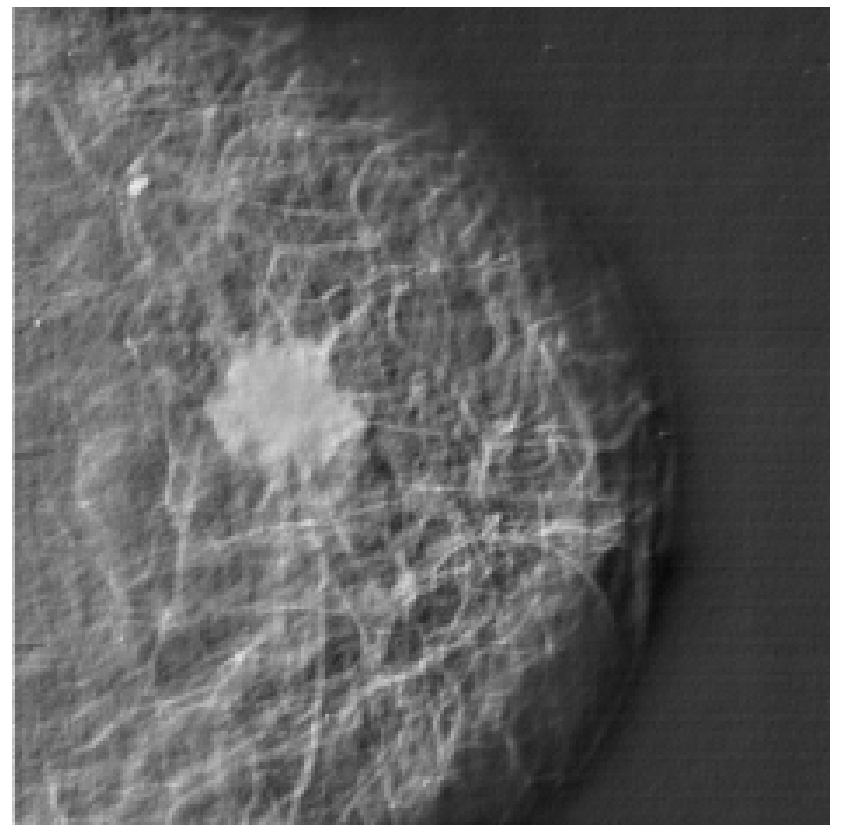

Figure 6. An enhanced image obtained by excluding the coefficients with orientation $\frac{\pi}{3}$ from processing with the same enhancement function as in Figure 5.

[5] A. Laine, J. Fan, and S. Schuler, "A framework for contrast enhancement by dyadic wavelet analysis," in Digital Mammography, A. G. Gale, S. M. Astley, D. R. Dance, and A. Y. Cairns, Eds., Amsterdam, The Netherlands: Elsevier, 1994, pp. 91-100.

[6] A. Laine, I. Koren, W. Yang, and F. Taylor, "A steerable dyadic wavelet transform and interval wavelets for enhancement of digital mammography," in Wavelet Applications II. H. H. Szu, Ed., Proc. SPIE, Orlando, FL, Apr. 1995, vol. 2491, pp. 736-749.

[7] W. T. Freeman and E. H. Adelson, "The design and use of steerable filters." IEEE Trans. Pattern Anal. Machine Intell., vol. PAMI-13, pp. 891-906, 1991.

[8] S. Mallat and S. Zhong, "Characterization of signals from multiscale edges," IEEE Trans. Pattern Anal. Machine Intell., vol. PAMI-14, pp. 710-732, 1992.

[9] P. Perona, "Deformable kernels for early vision," IEEE Trans. Pattern Anal. Machine Intell., vol. PAMI-17, pp. 488-499, 1995

[10] E. P. Simoncelli and W. T. Freeman, "The steerable pyramid: a flexible architecture for multi-scale derivative computation," in Proc. IEEE Int. Conf. Image Processing, Washington, D.C., Oct. 1995, vol. 3, pp. 444-447.

[11] E. P. Simoncelli, W. T. Freeman, E. H. Adelson, and D. J. Heeger, "Shiftable multiscale transforms," IEEE Trans. Inform. Theory, vol. IT-38, pp. 587-607, 1992.

[12] H. Greenspan, S. Belongie, R. Goodman, P. Perona, S. Rakshit, and C. H. Anderson, "Overcomplete steerable pyramid filters and rotation invariance," in Proc. IEEE Comput. Soc. Conf. Comput. Vision and Pattern Recogn., Seattle, Jun. 1994, pp. 237-244.

[13] A. Laine, J. Fan, and W. Yang, "Wavelets for contrast enhancement of digital mammography," IEEE Eng. Med. Biol. Mag., vol. 14, pp. 536-550, 1995. 